\title{
Pseudobulbar Affect: Burden of Illness in the USA
}

Jennifer Colamonico · Andrea Formella · Walter Bradley

To view enhanced content go to www.advancesintherapy.com

Received: June 28, 2012 / Published online: August 30, 2012

( ) The Author(s) 2012. This article is published with open access at Springerlink.com

\section{ABSTRACT}

Introduction: Pseudobulbar affect (PBA) is characterized by involuntary and uncontrollable laughing and/or crying episodes, occurring secondary to neurological disease or injury. The impact of PBA on social and occupational function, health status, quality of life (QOL), and quality of relationships (QOR) is not well studied.

Methods: This US survey conducted by Harris Interactive compared health status and daily function of patients with and without PBA.

J. Colamonico

Harris Interactive Inc., New York, New York, USA

A. Formella $(\bowtie)$

Avanir Pharmaceuticals, Inc., 20 Enterprise, Suite 200,

Aliso Viejo, California 92656, USA

e-mail: aformella@avanir.com

W. Bradley

Miller School of Medicine, University of Miami, Miami, Florida, USA

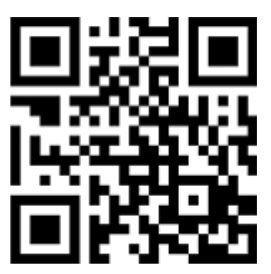

Enhanced content for Advances in Therapy articles is available on the journal web site: www.advancesintherapy.com
Eligible respondents were Harris Panel Online registrants previously diagnosed with stroke, multiple sclerosis, Parkinson's disease, Alzheimer's disease, traumatic brain injury, or amyotrophic lateral sclerosis, or primary, nonpaid caregivers for such patients who were too debilitated to participate. PBA was identified by a Center for Neurologic Study lability scale score of 13 or greater. Measures included the 36-item short form health survey (SF-36), the work productivity and impairment (WPAI) questionnaire, visual analog scales (VAS) for impact of PBA symptoms on QOL and QOR, and customized questions related to burden and impact of involuntary laughing/crying episodes on patients' lives. Survey responses were weighted to adjust for the relative proportion of the primary neurological conditions in the overall population and between group differences in patient age and gender. PBA and non-PBA group responses were compared using two-tailed $t$ tests adjusted for severity of the primary neurological conditions.

Results: The 1,052 respondents included 399 PBA group participants and 653 controls. The PBA group showed significantly worse scores versus non-PBA controls on component and summary SF-36 scores $(P<0.05$ for all), 
VAS scores $(P<0.05$ for both), and WPAI scores $(P<0.05)$. Among PBA group respondents, PBA contributed a great deal to or was the main cause of patients becoming housebound for $24 \%$ and being moved to supervised living placement for $9 \%$ of respondents.

Conclusion: PBA is associated with considerable burden incremental to that of the underlying neurological conditions, affecting QOL, QOR, health status, and social and occupational functioning.

Keywords: Burden of illness; Health status; Occupational function; Pseudobulbar affect; Quality of life; Social function

\section{INTRODUCTION}

Pseudobulbar affect (PBA) is a neurological disorder characterized by involuntary, sudden, and frequent episodes of laughing and/or crying that are typically out of proportion or incongruent to the underlying emotional state [1-4]. Although the etiology of PBA is not completely understood, it is strongly associated with neurological disease or injury that affects the frontal lobes and descending pathways to the brainstem, basis pontis, and cerebellum, and its symptoms appear to be similar across different patient populations [1-3]. PBA has been reported secondary to a wide variety of neurological conditions; available prevalence data suggest that neurological conditions commonly associated with PBA include Alzheimer's disease (AD) and other dementias, amyotrophic lateral sclerosis (ALS), multiple sclerosis (MS), Parkinson's disease (PD), stroke, and traumatic brain injury (TBI) $[2,3,5]$.

Partly owing to different preferences across physician specialties, a variety of terms have been used to describe syndromes of inappropriate laughing and/or crying, and this has contributed to inconsistency and confusion in the literature. PBA is used in this paper; however, other commonly used terms have included pathological laughing and crying, affective lability, emotional incontinence, emotional lability, and involuntary emotional expression disorder.

PBA may cause severe distress, embarrassment, and social disability for patients [1, 3, 6-9]; however, the burden of illness associated with PBA is not well characterized. Studies in populations with stroke, PD, and other movement disorders have shown that patients with PBA or similarly described symptoms of inappropriate laughing and/or crying have a greater incidence of depression [10, 11] and decreased executive function [12], sexual function [13], and ability to perform activities of daily living (ADL) [14] compared with patients with the same underlying neurological disorder but without PBA symptoms.

Patients can be screened for PBA using the Center for Neurologic Study lability scale (CNS-LS). The CNS-LS is the first self-report measure of PBA to be described, and has been validated in ALS [15] and MS [16] patients. The CNS-LS consists of subscales for laughter (four items) and for tearfulness (three items), with each item scored on a 5-point scale (1, applies never; 5 , applies most of the time) for a total score ranging from 7 (no symptoms) to 35 (maximum). In patients with ALS $(n=99)$, a CNS-LS score of 13 or greater correctly predicted neurologists' diagnoses of PBA for $82 \%$ of patients (sensitivity of 0.84 ; specificity of 0.81 ); the CNS-LS also showed good test-retest reliability (0.88) and internal consistency (Cronbach's $\alpha$ coefficient 0.87) [15]. In patients with MS $(n=90)$, a CNS-LS score of 13 or greater correctly predicted physicians' diagnoses of PBA for 78\% of patients (sensitivity of 0.96 ; specificity of 0.55 ), and a CNS-LS score of 17 or greater correctly predicted $89 \%$ of physicians' diagnoses (sensitivity of 0.94 ; specificity of 0.83 ) [16]. 
The authors have recently published the results of a survey conducted using the CNS-LS to estimate the prevalence of PBA in the USA across six commonly associated underlying conditions: AD and other dementias, ALS, MS, PD, stroke, and TBI [5]. Adopting a conservative CNS-LS cutoff score of 21 or greater yielded an overall prevalence of $9.4 \%$, which translated to 500,000 to 2 million individuals in the USA with PBA. With a lower CNS-LS threshold of 13 or greater, which helps identify less severely affected patients with a reasonable sensitivity and specificity, the prevalence was even higher (37.5\%), or a total of 1.8-7.1 million individuals.

The authors report the results of a follow-on survey to estimate the impact, or burden of illness, of PBA on general health status and overall functionality by comparing measures of general health, social and occupational function, quality of life (QOL), and other measures in patients and caregivers for patients with AD, ALS, MS, PD, stroke, and TBI with and without PBA symptoms.

\section{MATERIALS AND METHODS}

This study was commissioned by Avanir Pharmaceuticals and conducted online by Harris Interactive $^{\circledR}$ (HI, New York, New York, USA) a market research firm specializing in public opinion and consumer surveys. The survey was designed and directed by Avanir Pharmaceuticals and statistical analysis and study design specialists from HI and Cerner Health Insights. The survey sample included patients with PBA symptoms or primary, nonpaid caregivers of patients with PBA symptoms to answer for patients unable to do so themselves, and thus ensure participation of more severely incapacitated patients. For purposes of the study, PBA symptoms were defined as a CNS-LS score of 13 or greater based on the minimum CNS-LS baseline score required for inclusion in recent PBA clinical trials [17-19]. The online survey was fielded between January 27, 2006 and March 31, 2006. The average survey length was 18 minutes.

\section{Survey Sample}

Survey participants were recruited in two waves. To recruit the PBA group, invitations were first sent to 857 members of the Harris Panel Online (HPOL) sample, who had voluntarily agreed to participate in various $\mathrm{HI}$ online research surveys, had participated in the previous PBA prevalence survey, and had scored 13 or more on the CNS-LS in that survey, suggestive of PBA. To complete recruitment of the PBA group and to recruit the controls, invitations were then sent to a nationally representative sample of more than 6 million US adults (aged 18 years and over), registered in the HPOL, in a similar fashion to the recruitment procedure for the previous PBA prevalence survey [5]. At the time of recruitment, the HPOL included approximately 1.5 million individuals who had been screened by HI by means of a checklist for the presence of chronic illnesses. HPOL panel members previously identified by $\mathrm{HI}$ as either having AD, ALS, MS, PD, and stroke or being primary, nonpaid caregivers for a household member with one of these conditions were sent online invitations to participate in a survey about their "thoughts on and experiences with some important healthcare-related topics" [5]. To enhance the representativeness of the total sample, invitations were also sent to a randomly selected national sample from the general HPOL. Recruitment quotas were set for each underlying condition in order to have a large enough sample of each disease group for analysis.

The HI chronic illness screener instrument did not include patients with TBI [20]. To identify and recruit patients with TBI, survey invitations were 
sent to panel members with chronic headaches, posttraumatic stress disorder, or general disability. The survey asked these members whether they had been diagnosed with TBI by a physician or other healthcare professional, defined as a head injury requiring hospitalization, which resulted from being knocked unconscious, having a skull fracture, or having other brain injury. Because US data show that only approximately $16 \%$ of TBI survivors each year are hospitalized for their injury, the TBI patients participating in this study probably represented a subset of relatively severe cases [20].

The survey invitations were identical for all invitees, i.e., the invitations that were sent to HPOL members who had participated in the previous prevalence survey did not reference that survey or the participants' previous participation. Invitations included a unique log-in name and password to confirm that the person responding was the selected participant. Selection of the ultimate sample for each primary condition was designed to include a mix of responses from patients and caregivers, with target proportions of two-thirds patients and one-third caregivers in both the PBA and control groups. Numeric quotas were assigned to each disease group and proportion of respondents with PBA and without PBA within each disease group. The online program assigned respondents into each quota group based on survey logic.

\section{Identification of Patients with PBA and Matched Controls}

Eligible respondents (those confirmed to be patients or primary, nonpaid caregivers for patients with one of the six designated conditions) completed an online CNS-LS $[15,16]$. Patients and caregivers were asked the same screening questions, with caregivers answering based on their observations of the patient.
Respondents with a CNS-LS score of 13 or greater were assigned to the PBA group; respondents with a CNS-LS score less than 13 were assigned to the control group. The control (non-PBA) participants were included to discern the burden of PBA incremental to other problems stemming from the primary neurological disease. Once the survey program assigned each respondent to either the PBA or non-PBA group, respondents continued through the remainder of the survey.

\section{Burden of Illness Measures}

In order to measure various aspects of burden of illness in PBA group respondents and controls, a series of medically validated scales was administered, including the 36-item short form health survey (SF-36), the work productivity and activity impairment (WPAI) questionnaire: general health V2.0, the screen for caregiver burden (SCB), the Center for Epidemiologic Studies depression scale 10-item short form (CES-D10), and visual analog scales (VAS) for QOL and quality of relationships (QOR).

The SF-36 health survey is a 36-question self-reported measure that rates general health and well-being across eight domains, which are summarized into physical and mental health component measures. Scores for each domain and the summary physical and mental health component measures are transformed into scores of $0-100$ ( 0 , worst health; 100 , best health) [21]. The WPAI questionnaire measures employment and rates of absenteeism, reduction in work productivity, and impairment in regular daily activities other than working, during the previous 7 days [22]. The SCB probes the prevalence and associated distress of 25 potentially negative experiences of caregiving [23]. The SCB yields two scores. The objective score (0-25) counts the presence of experiences from a list of 25 burdens, such as "I am upset 
that I cannot communicate with my patient," "I feel so alone, as if I have the world on my shoulders," and "I am embarrassed to take my patient out for fear he/she will do something bad." The subjective score (25-100) indicates the degree of distress associated with each item (1, "no occurrence" or "occurrence, but no distress;" 2, "mild distress;" 3, "moderate distress;" or 4, "severe distress"). The CES-D10 scale is a screening test for depression with each item scored from 0 to 3 (scoring range of $0-30$ ), and with a validated cutoff score of 10 or greater for clinically meaningful depressive symptoms [24]. VAS for QOL and QOR asked patients to indicate "the degree to which uncontrollable laughter, tearfulness, or anger" had affected the overall QOL and the overall QOR during the past week, by making a mark on a horizontal line with anchors of " 0 , not at all" on the left and "100, continuously" on the right.

In addition to the above scales, specific questions were asked to assess the emotional state of the patients over the past week, the frequency, burdensomeness, and emotional impact of involuntary laughing and/or crying episodes, and the degree to which these episodes disrupted specific life activities. For example, respondents were asked the degree to which episodes of involuntary laughter or crying interfered with activities such as spending time

Table 1 Summary of scales: who responded and from which perspective

\begin{tabular}{|c|c|c|}
\hline Scale & Caregiver perspective & Patient perspective \\
\hline \multicolumn{3}{|l|}{ Completed by both the PBA and control group respondents } \\
\hline CNS-LS & As patient proxy & Self-rating \\
\hline Disease severity & $\begin{array}{l}\text { Caregiver opinion of } \\
\text { patient's disease severity }\end{array}$ & Self-rating \\
\hline SF-36 & As patient proxy & Self-rating \\
\hline CES-D10 & Caregiver self-rating & Self-rating \\
\hline WPAI questionnaire & Caregiver self-rating & Self-rating \\
\hline Caregiver burden & Caregiver self-rating & ND \\
\hline VAS QOL/QOR & ND & Self-rating \\
\hline Customized questions related to negative emotional feelings & $\begin{array}{l}\text { Caregiver opinion of } \\
\text { patient's feelings }\end{array}$ & Self-rating \\
\hline \multicolumn{3}{|l|}{ Completed by only PBA group respondents } \\
\hline $\begin{array}{l}\text { Customized questions related to laughing/crying episode frequency, } \\
\text { severity, and impact }\end{array}$ & $\begin{array}{l}\text { Caregivers in consideration } \\
\text { of patients' episodes }^{\mathrm{a}}\end{array}$ & Self-rating ${ }^{\mathrm{a}}$ \\
\hline
\end{tabular}

CES-D10 Center for Epidemiologic Studies depression scale 10-item short form, CNS-LS Center for Neurologic Study lability scale, $N D$ not determined, $P B A$ pseudobulbar affect, $Q O L$ quality of life, $Q O R$ quality of relationships, $S F-3636-$ item short form health survey, VAS visual analog scale, WPAI work productivity and activity impairment questionnaire: general health V2.0

${ }^{a}$ Customized questions related to laughing and crying episodes were only answered by PBA group patients and caregivers who also answered "yes" to the question, "Have you [has the patient you care for] experienced involuntary episodes of crying and/or laughing that were exaggerated or even contrary to how you [your patient] felt at the time?” 
with friends and family, dining out, or going to the movies; the degree to which episodes caused them to feel frustrated, isolated, embarrassed, or were a problem for those around them, and the extent to which episodes contributed to life situations such as becoming housebound, losing a job, severing close relationships, or moving to supervised living.

Both patients and caregivers in the PBA and control groups completed the SF-36, with questions for caregivers adjusted to instruct them to respond on behalf of the patient (caregivers responded as patient proxies). Patients and caregivers rated themselves individually on the CES-D10 scale and the WPAI questionnaire (caregivers answered on their own behalf). Only patients (caregivers were not used as patient proxies) responded to the VAS QOL and QOR questions. Only caregivers responded to the SCB. Only the PBA group patients and caregivers (no controls) answered the customized questions related to the frequency, burdensomeness, and impact of PBA episodes, with caregivers answering these questions on behalf of their patients. Table 1 provides a summary of the scales and respondents.

\section{Control for Underlying Disease Severity}

In order to account for the impact of potential differences in underlying disease severity on results, respondents were asked to self-rate the severity of their/their patient's underlying disease in response to a single question: "In your opinion, how would you classify the severity of your/your patient's [condition]?" The given choices were mild, moderate, or severe. Based on the responses to this question, weighting multipliers were added to survey responses within each primary disease group of PBA and control respondents to adjust for disease severity.

As a sensitivity measure, respondents were also administered, established, and validated severity scales designed for individual diseases, including the ALS functional rating scale (ALSFRS) for ALS patients [25], the Schwab and England ADL scale [26] for MS, PD, stroke, and TBI, and the instrumental ADL (IADL) scale [27] for $\mathrm{AD}$ patients. The respondent scores on the Schwab and England and IADL scales were then correlated with the respondent self-ratings of disease severity (mild, moderate, or severe) to validate the self-ratings; correlations were not done for the ALS group due to the multiple outputs of the ALS-FRS as well as the small sample of ALS patients.

\section{Demographic Weighting}

To adjust for demographic differences between the PBA and control groups, weighting multipliers were applied to PBA and control respondent data by disease group to make them more similar to each other in terms of age and gender (when sample size was sufficiently large) to minimize variation in responses that might be attributable to these demographic characteristics. This was not possible for the AD and ALS disease groups. The number of $\mathrm{AD}$ and other dementias patient respondents was too small to apply an adjustment because, due to the disabling nature of the disease, $90 \%$ of the $\mathrm{AD}$ and other dementias respondents were caregivers. The number of ALS patient respondents was also too small to apply an adjustment due to difficulties with recruitment stemming from the low prevalence of this condition in the overall population. All "unweighted" respondents were given a demographic weight of 1.0.

To adjust for the relative proportions of the six underlying conditions within the total US population (including the estimated prevalence of PBA within each condition), and thus increase generalizability to the total US patient population, additional post weights were 
Table 2 Disease group proportions in study population and weighting to relative US disease prevalence

\begin{tabular}{lcc}
\hline Disease group & Study population proportions ${ }^{\mathrm{a}}(\%)$ & Weighted proportions $^{\mathrm{b}}(\%)$ \\
\hline ALS & 3.7 & 0.2 \\
AD/dementia & 17.1 & 36.7 \\
MS & 21.6 & 2.7 \\
PD & 19.0 & 3.4 \\
Stroke & 21.6 & 20.4 \\
TBI & 17.0 & 36.6 \\
\hline
\end{tabular}

$A D$ Alzheimer's disease, $A L S$ amyotrophic lateral sclerosis, $C N S$ - $L S$ Center for Neurologic Study lability scale, $M S$ multiple sclerosis, $P B A$ pseudobulbar affect, $P D$ Parkinson's disease, $T B I$ traumatic brain injury

${ }^{a}$ Relative proportion of respondents with each primary disease in the current survey

${ }^{\mathrm{b}}$ The weighted proportions represent the estimated relative proportions of patients with PBA among underlying illnesses in the US population. These were calculated by taking the estimated number of patients in the USA with each of the underlying diseases, and then estimating the number of patients with PBA symptoms (CNS-LS $\geq 13$ ) within each disease state and dividing by the total number of US patients estimated to have PBA (number of patients with CNS-LS $\geq 13$ using prevalence source 1 [5])

applied to all outcomes reported for the total study population across $\mathrm{PBA} / \mathrm{control}$ and disease subgroups (Table 2) [5].

\section{Margin of Sampling Error}

In a random probability sample of respondents, study results are subject to a margin of sampling error, which is calculated using the size of the sample. Although the sample in this study was not a random probability sample in its true sense, because any Internet panel is made up of those who self-select to join, margins of error were calculated and are provided for general reference in interpreting the survey results. Assuming a 95\% confidence interval for statistical testing across the board, the margin of error was $\pm 4.9 \%$ for the total PBA group and $\pm 3.8 \%$ for the non-PBA group. For individual disease states within the PBA group, the margin of error was $18.9 \%$ for the ALS group and ranged from $\pm 11.1 \%$ to $12 \%$ for the other disease states; for the non-PBA group, the margin of error was $30.2 \%$ for the ALS group and ranged from $\pm 8 \%$ to $9.6 \%$ for the others.

\section{Significance Tests}

Two group $t$ tests were conducted to test for mean differences in study measures between the PBA and control groups and within each disease state. All significance testing was performed at the $95 \%$ confidence interval, two tailed. These tests were performed after all other weighting (as described above) was applied to the data. The severity weights were used to validate the statistical significance of differences, and all reported $P$ values in the article reflect adjustment for disease severity; however, numerical results reported reflect only the demographic weighting as described above.

\section{RESULTS}

\section{Study Population}

Of 857 patients/caregivers of patients with a CNS-LS score of 13 or greater from the initial HPOL prevalence survey [5] who were recontacted in the first wave of recruitment for this survey, 599 (70\%) responded, of whom 
453 were still qualified to participate (had the diagnosis in question or remained the primary, nonpaid caregivers of such a patient) and completed the CNS-LS screener. A total of 341 (75\%) of these 453 still had a CNS-LS score of 13 or greater, and 285 of these participated in the present survey as part of the PBA group; the remaining 56 respondents with a CNS-LS score of 13 or greater did not participate, as their disease quotas were already full. For the remaining recruitment, a random sample ( $n=27,696)$ of the HPOL database previously identified as having ALS, AD or other dementias, MS, PD, stroke, or symptoms suggestive of TBI were invited to participate. Of the 9,283 (34.5\%) individuals who responded, 2,499 (26.9\%) were found eligible (patient or primary, nonpaid caregiver with one of the disease states of interest and completed the online CNS-LS screen). Of these 2,499 individuals, 767 participated in the survey and 1,732 were qualified but did not participate, as the quotas for their disease states were already full.
Therefore, in total 1,052 respondents completed the survey; 399 (37.9\%) fulfilled the criteria for PBA (CNS-LS score $\geq 13$ ) and $653(62.1 \%)$ comprised the non-PBA (CNS-LS score $<13$ ) control group (Table 3 ). In the PBA group, $66.4 \%$ were patients and $33.6 \%$ were caregivers, and in the control group, $63.3 \%$ were patients and $36.7 \%$ were caregivers (Table 3). Recruitment did not meet the quota for the ALS group. The goal of achieving proportions of two-thirds patients and one-third caregivers was achieved for most diseases in the PBA group, but not for the control group, most notably for ALS, which comprised only 11 controls including four patients (36\%) and seven caregivers (64\%), and $\mathrm{AD}$ and other dementias, which comprised $8 \%$ patients and $92 \%$ caregivers (Table 3 ).

Demographic characteristics are provided in Table 4 . The mean patient age was 55.5 years in the PBA group and 61.8 years in the control group; patients with TBI, ALS, and MS were generally younger than those with stroke, PD, and AD. Fifty-eight percent of the PBA group

Table 3 Survey respondents by primary condition ${ }^{a}$

\begin{tabular}{lcccccc}
\hline Primary condition & \multicolumn{3}{c}{ PBA group $^{\mathrm{b}}$} & \multicolumn{3}{c}{ Control group $^{\mathrm{c}}$} \\
\cline { 2 - 6 } & Patients & Caregivers & Totals & Patients & Caregivers & Totals \\
& $n(\%)$ & $n(\%)$ & $n$ & $n(\%)$ & $n(\%)$ & $n$ \\
\hline ALS & $19(68)$ & $9(32)$ & 28 & $4(36)$ & $7(64)$ & 11 \\
AD/dementia & $42(63)$ & $25(37)$ & 67 & $9(8)$ & $104(92)$ & 113 \\
MS & $53(68)$ & $25(32)$ & 78 & $120(81)$ & $29(19)$ & 149 \\
PD & $50(67)$ & $25(33)$ & 75 & $73(58)$ & $52(42)$ & 125 \\
Stroke & $51(67)$ & $25(33)$ & 76 & $117(78)$ & $34(22)$ & 151 \\
TBI & $50(67)$ & $25(33)$ & 75 & $90(87)$ & $14(13)$ & 104 \\
Total & $265(66)$ & $134(34)$ & 399 & $413(63)$ & $240(37)$ & 653 \\
\hline
\end{tabular}

$A D$ Alzheimer's disease, $A L S$ amyotrophic lateral sclerosis, $C N S$ - $L S$ Center for Neurologic Study lability scale, $M S$ multiple sclerosis, $P B A$ pseudobulbar affect, $P D$ Parkinson's disease, $T B I$ traumatic brain injury

${ }^{a}$ Respondents consisted of patients or primary, nonpaid caregivers of patients

b The PBA group was defined by a score of 13 or greater on the CNS-LS

${ }^{\mathrm{c}}$ Controls were patients (or caregivers of a patient) who scored less than 13 on the CNS-LS 
patients and $57 \%$ of the control group patients were women, with the lowest percentages in the ALS and TBI groups and the highest percentage in the MS group. Fewer PBA group patients (7\%) than control patients (21\%) were living independently; however, most patients lived with their caregivers (74\% PBA group; 65\% non-PBA controls). Overall, most caregivers were spouses (34\% PBA group; $44 \%$ non-PBA controls) or children (38\% PBA group; $34 \%$ nonPBA controls) of the patients for whom they provided care.

The mean (median; range) baseline CNS-LS scores were $18.75(18 ; 13-35)$ in the PBA group and $9.17(9 ; 7-12)$ in the control group. Of the PBA group respondents, $30.4 \%$ had a CNS-LS score of 21 or greater, indicative of more moderate to severe PBA. PBA group patients $(72 \%)$ were significantly more likely than controls $(45 \%)$ to have been diagnosed with any of the following psychiatric conditions along with their primary neurological condition: depression (52\% vs. 28\%), anxiety/panic attacks (43\% vs. 17\%), bipolar disorder (13\% vs. $4 \%)$, posttraumatic stress disorder $(20 \%$ vs. $11 \%)$, psychotic disorder (9\% vs. $1 \%)$, or schizophrenia/delusional disorder (5\% vs. $2 \%$ ) $(P<0.05$ for overall prevalence of a psychiatric diagnosis and for each diagnosis). The degree to which the greater prevalence of these diagnoses in the PBA group may represent a misdiagnosis of PBA symptoms is unclear and is not able to be determined from the survey.

While the majority of respondents assessed the severity of their/their patients' primary neurological disease as mild or moderate (Table 4), significantly more PBA respondents in the PD (13\% PBA vs. $3 \%$ controls) and stroke (18\% PBA vs. 7\% controls) groups rated their/their patients' condition as severe ( $P<0.05$ for both). On the validated disease-specific severity scales, PBA group respondents with MS, PD, stroke, and TBI scored significantly lower (indicating greater severity) on the Schwab and England ADL scale than non-PBA control respondents with the same conditions $(P<0.05)$. Among patients with $\mathrm{AD}$, mean scores on the IADL scale were similar between the PBA and control group patients (15.0 and 14.4, respectively). The sample size of patients with ALS $(n=48)$ was too small to assess for significance between the PBA and control groups on the ALS-FRS; however, no clear numerical differences were observed in the various components of this scale (bulbar symptoms, upper and lower limbs, and breathing).

The respondent self-ratings of global disease severity showed significant correlation ( $P<0.01$; Pearson product-moment correlation) to the disease-specific scales for $\mathrm{AD}(r=-0.66)$, MS $(r=0.66)$, PD $(r=0.54)$, and stroke $(r=0.32)$, suggesting that the respondent self-ratings were a valid measure of disease severity. Although the correlation for TBI $(r=0.11)$ was not significant, this may be reflective of low variation in responses for the global self-assessment in this group ( $>60 \%$ said moderate).

\section{General Health Status}

Respondents screening positive for PBA (CNS-LS score $\geq 13$ ) had significantly lower (worse) scores on all eight domains, as well as the physical and mental component scores, of the SF-36 compared with respondents in the non-PBA control group (CNS-LS score <13) (Fig. 1). The adjusted mean physical component summary and mental component summary scores were 35.0 and 34.4, respectively, for the PBA group respondents, compared with 39.1 and 42.5, respectively, for the non-PBA controls $(P<0.05)$ (Fig. 1). The between-group differences were generally larger for the mental health domain items than for the physical health domain items, 


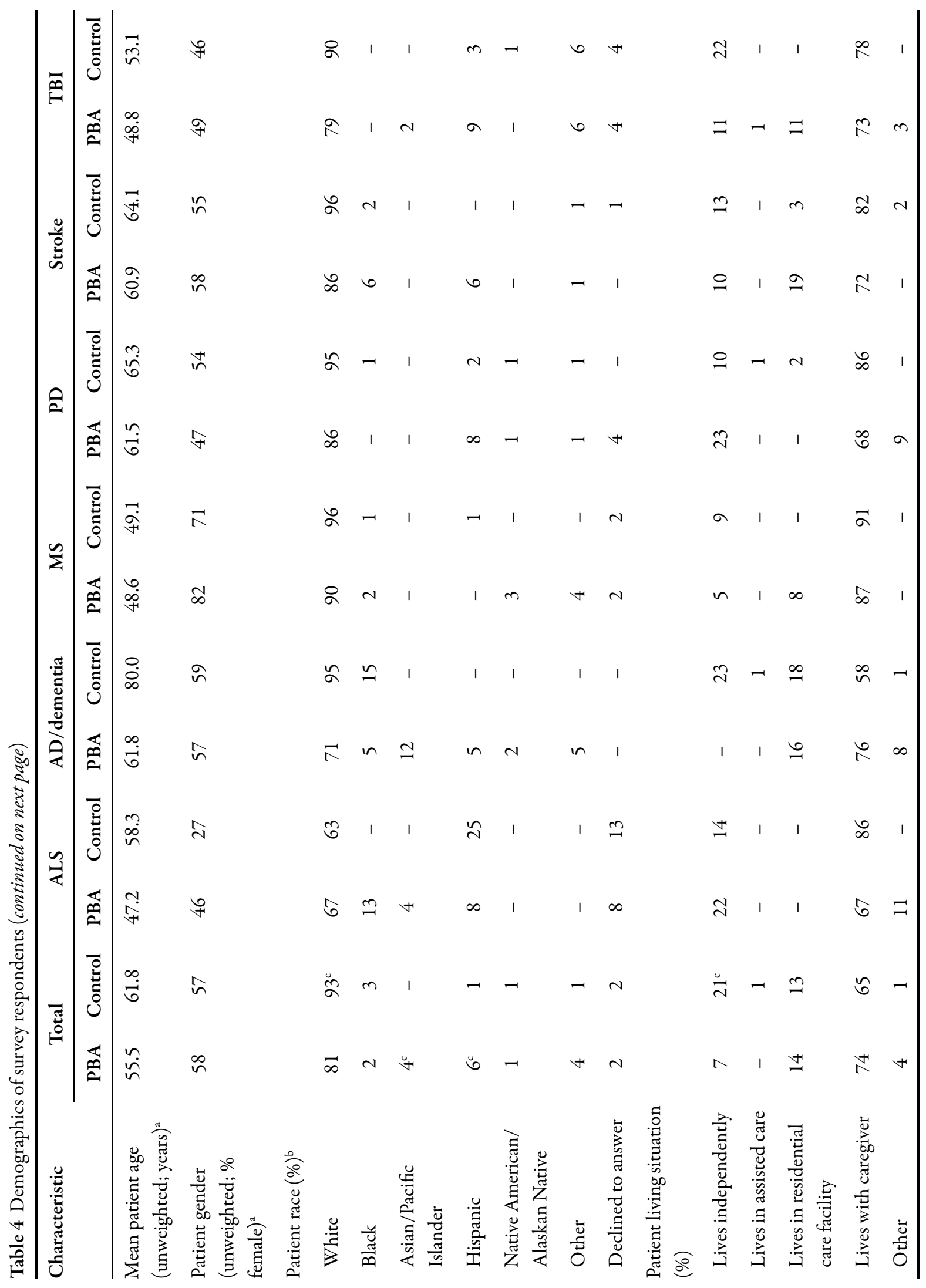




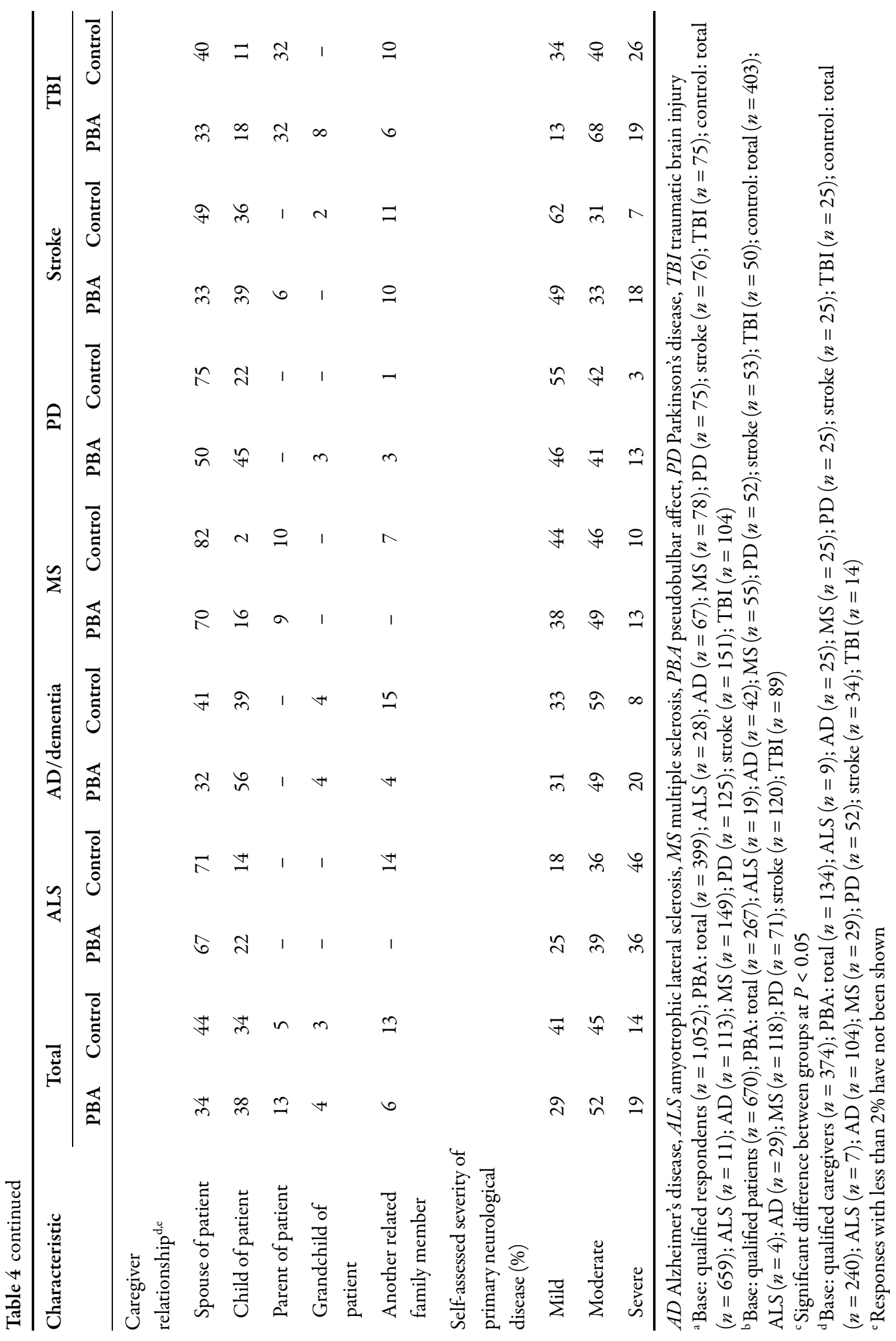


a

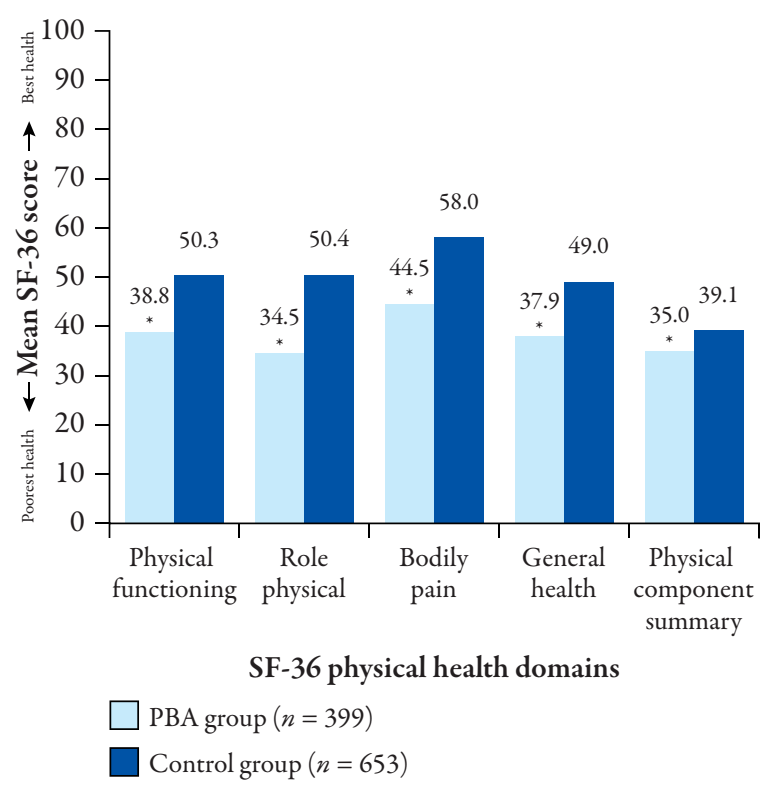

b

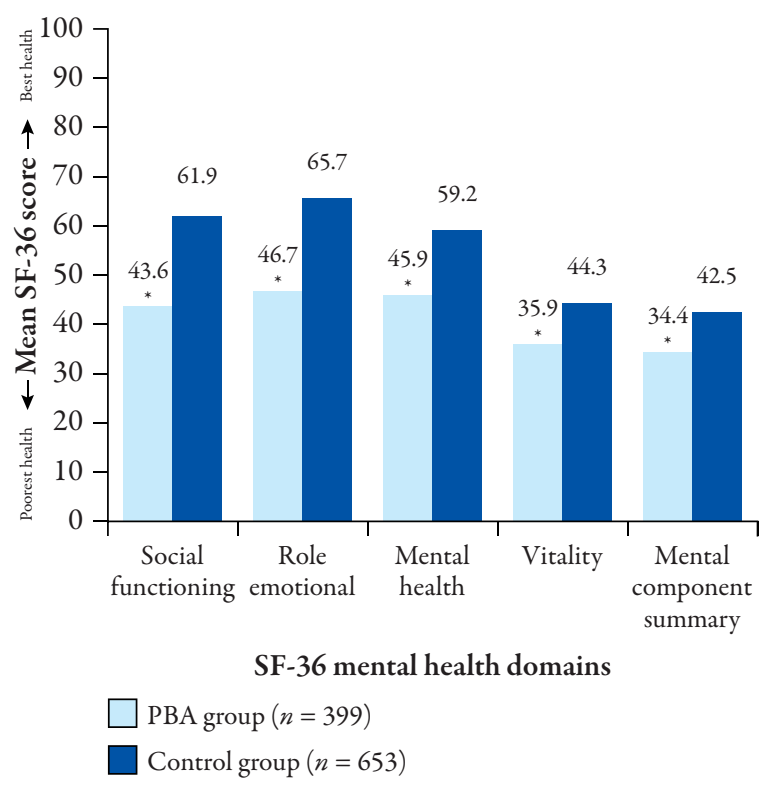

Fig. 1 SF-36 mean physical (a) and mental health (b) domain and component summary scores. The mean scores are adjusted for demographic differences; $P$ values are calculated with adjustment for underlying disease severity. ${ }^{*} P<0.05$ independent samples two-tailed $t$ test. $P B A$ pseudobulbar affect, $S F-3636$-item short form health survey

as well as the mental component summary; the PBA group also scored significantly worse than the non-PBA controls within each disease group except ALS $(P<0.05)$.

\section{Impact of PBA Symptoms on QOL and QOR}

VAS measurements demonstrated a significantly larger negative impact of uncontrollable laughter, tearfulness, or anger on PBA group patients' overall QOL and QOR during the past week (37.9 and 37.0, respectively) versus controls (12.3 and 10.4, respectively; $P<0.05$ [Fig. 2]). The differences in VAS QOL and QOR scores were also significant for all disease subpopulations $(P<0.05)$ with the exception of the QOL measure in ALS patients $(n=23)$, among whom the mean scores were 46.3 for the PBA group and 15.3 for the controls; however, the number of ALS control patients $(n=4)$ was too small to provide a reliable result.
The QOL and QOR scores correlated highly with the mental component summary of the SF-36 ( $r=-0.55$ and -0.61 , respectively), supporting the hypothesis that the VAS and the mental domains of the SF-36 are measuring a similar construct. QOL and QOR were not highly correlated with the physical component summary of the SF-36 ( $r=-0.18$ and -0.14 , respectively), thus not supporting construct validity for the QOL and QOR measuring a physical construct.

\section{The WPAI Questionnaire}

The PBA group patients and caregivers were less likely to be employed for pay, and experienced greater work and activity impairment than the control group patients and caregivers (Table 5). Among patients, significant differences over the previous 7 days were observed between the PBA 


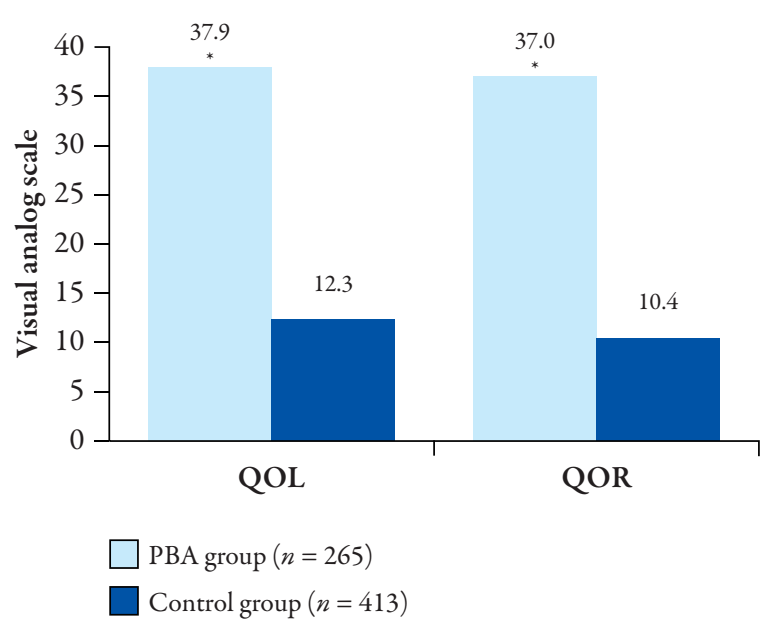

Fig. 2 Adjusted mean PBA and non-PBA control group visual analog scale $(0-100)$ scores. Patients were asked to mark on horizontal lines with anchors of "not at all" at the leftmost position ("0") and "continuously" at the rightmost position ("100") how much episodes of uncontrollable laughter, tearfulness, and anger have affected their overall QOL and their overall QOR with others during the past week. Mean scores are adjusted for demographic differences; $P$ values are calculated with adjustment for underlying disease severity. ${ }^{*} P<0.05$ independent samples two-tailed $t$ test. Patients rated QOL and QOR on their own behalf (caregivers did not serve as patient proxies for these measures). $P B A$ pseudobulbar affect, $Q O L$ quality of life, $Q O R$ quality of relationships

and non-PBA control groups in the rate of workplace absenteeism $(P<0.05)$, overall work impairment/productivity loss $(P=0.05)$, and overall impairment in daily activities outside of work $(P<0.05)$ due to the patients' health problems. Among caregivers, significant differences were observed between the PBA and non-PBA control groups in the degree to which their patients' health problems affected caregiver productivity while at work (presenteeism) and overall work impairment/productivity loss. However, there were no significant differences between the PBA and control group caregivers in terms of employment status, workplace absenteeism, and nonwork activity impairment.

\section{Depression: Patient Respondents}

Most patients in the PBA group and over half in the control group had significant depressive symptoms as determined by a CES-D10 scale score of 10 or greater (87\% PBA group vs. $56 \%$ controls; $P<0.05)$. Within the individual disease categories, $74 \%$ of the PBA group patients with ALS and $82-89 \%$ in the other disease categories exceeded this CES-D10 threshold versus a range of $38-75 \%$ among controls across all disease categories. The prevalence of depressive symptoms (CES-D10 scale score $\geq 10$ ) was significantly higher in the PBA versus the control group patients in the MS, PD, stroke, and TBI disease groups $(P<0.05)$. While rates of depressive symptoms were also higher among the PBA group patients with AD and ALS than among controls with the same diseases, the differences were less pronounced and the disease subgroups were too small for these differences to reach statistical significance.

Given the high frequency of depressive symptoms observed among PBA group patients, and previous research showing that the presence of such symptoms may significantly affect SF-36 scores [28], a post hoc multivariate analysis of variance (MANOVA) was performed to determine the impact of PBA group assignment, depression, and their interaction on SF-36 scores. This approach compares the mean SF-36 domain score for each of the four types of respondents: PBA group respondents with depression; PBA group respondents without depression; control group respondents with depression; and control group respondents without depression. In addition, the analysis takes into account the fact that the same variables (PBA and depression) are being tested many times. Only patient respondents were included in this analysis as caregiver respondents answered the CES-D10 questions on their own behalf 
Table 5 Mean work productivity and activity impairment questionnaire scores ${ }^{\mathrm{a}}$

\begin{tabular}{|c|c|c|c|c|}
\hline \multirow[t]{2}{*}{ Parameter } & \multicolumn{2}{|c|}{ Patients $(N=678)$} & \multicolumn{2}{|c|}{ Caregivers $(N=374)$} \\
\hline & $\begin{array}{c}\text { PBA } \\
(n=265)\end{array}$ & $\begin{array}{l}\text { Controls } \\
(n=413)\end{array}$ & $\begin{array}{c}\text { PBA } \\
(n=134)\end{array}$ & $\begin{array}{l}\text { Controls } \\
(n=240)\end{array}$ \\
\hline Employed/work for pay & 34 & 42 & 45 & 51 \\
\hline Workplace absenteeism & $24^{\mathrm{b}}$ & 12 & 10 & 9 \\
\hline Workplace presenteeism (impairment while at work) & 39 & 33 & $44^{\mathrm{b}}$ & 27 \\
\hline Work productivity loss (overall work impairment) & $50^{\mathrm{b}}$ & 38 & $48^{b}$ & 32 \\
\hline Overall activity impairment (daily activities other than work) & $58^{\mathrm{b}}$ & 41 & 47 & 42 \\
\hline \multicolumn{5}{|c|}{$\begin{array}{l}\text { a'Other than employment rate, which is expressed as a percentage of respondents, scores are expressed as impairment } \\
\text { percentages, with higher numbers denoting greater impairment; absenteeism: percentage of work time missed due to your/ } \\
\text { your patient's health problem; presenteeism: percentage of impairment while working due to your/your patient's health } \\
\text { problem; overall work impairment: percentage of overall work impairment (absenteeism plus presenteeism); overall activity } \\
\text { impairment: percentage of activity impairment other than work due to your/your patient's health problem } \\
\text { b Significant difference at } P<0.05 \text {, tested with disease severity adjustments; work productivity score (combination of } \\
\text { absenteeism and reduced work productivity) }\end{array}$} \\
\hline
\end{tabular}

instead of as patient proxies. The MANOVA analysis demonstrated a significant main effect of depression (CES-D $\geq 10$ ) on all SF-36 domains, and of PBA group assignment (CNS-LS $\geq 13$ ) on all SF-36 domains except bodily pain and the physical component summary. An interaction of depression and PBA group assignment was seen for the SF-36 domains of role physical, role emotional, mental health, and the mental component summary with depression reducing the impact of PBA group assignment on these domains (Table 6).

\section{Depression: Caregiver Respondents}

Depressive symptoms (CES-D10 scale score $\geq 10$ ) were also present in most caregivers and occurred with similar overall frequency in the PBA and control groups (73\% and $71 \%$, respectively). Within disease subgroups, significantly more control group caregivers of patients with $\mathrm{AD}$ and other dementias experienced depressive symptoms (79\%) compared with their PBA group counterparts (56\%), whereas significantly more PBA group caregivers for patients with PD (68\%) and TBI (91\%) experienced depressive symptoms compared with controls (33\% and $43 \%$, respectively; $P<0.05$ for all).

\section{Screen for Caregiver Burden}

Mean total scores on the SCB suggested that the overall burden of caring for patients in the PBA group was slightly but significantly higher than for the control group. Both the objective (prevalence) and subjective (distress) scores were higher among caregivers in the PBA group (13.7 and 41.5, respectively; $P=0.05)$ compared with the control group (10.2 and 37.6, respectively; $P=0.05$ ).

\section{Impact on Feelings and Activities}

Significantly higher percentages of patients (including caregivers rating as patient proxies) in the PBA group than in the non-PBA control group reported negative emotional feelings in 
Table 6 Impact of PBA group assignment and depression ${ }^{a}$ on SF-36 scores; MANOVA results

\begin{tabular}{|c|c|c|c|c|}
\hline SF-36 domain & $\begin{array}{c}\text { PBA } \\
(\mathrm{CNS}-\mathrm{LS} \geq 13)^{\mathrm{b}, \mathrm{c}}\end{array}$ & $\begin{array}{c}\text { Depression } \\
(\mathrm{CES}-\mathrm{D} 10 \geq 10)^{\mathrm{b}, \mathrm{d}}\end{array}$ & Interaction $^{\mathrm{b}, \mathrm{e}}$ & Direction of PBA impact ${ }^{f}$ \\
\hline Physical functioning & 4.7, 0.031, Yes & $40.8,<0.0001$, Yes & $0.2,0.651$, No & $\begin{array}{l}\text { Reduces (depression does } \\
\text { not change PBA impact) }\end{array}$ \\
\hline Role physical & $10.5,0.001$, Yes & $57.7,<0.0001$, Yes & $11.5,0.001$, Yes & $\begin{array}{l}\text { Reduces (depression lessens } \\
\text { impact) }\end{array}$ \\
\hline Bodily pain & $1.9,0.169$, No & $73.6,<0.0001$, Yes & $1.3,0.259, \mathrm{No}$ & No impact \\
\hline General health & $9.7,0.002$, Yes & $51.8,<0.0001$, Yes & $0.7,0.393$, No & $\begin{array}{l}\text { Reduces (depression does } \\
\text { not change PBA impact) }\end{array}$ \\
\hline Vitality & 5.7, 0.017, Yes & $115.9,<0.0001$, Yes & $1.5,0.228$, No & $\begin{array}{l}\text { Reduces (depression does } \\
\text { not change PBA impact) }\end{array}$ \\
\hline Social functioning & $22.5,<0.0001$, Yes & $87.9,<0.0001$, Yes & $3.3,0.07$, No & $\begin{array}{l}\text { Reduces (depression does } \\
\text { not change PBA impact) }\end{array}$ \\
\hline Role emotional & $34.3,<0.0001$, Yes & $45.6,<0.0001$, Yes & 12.2, 0.001, Yes & $\begin{array}{l}\text { Reduces (depression lessens } \\
\text { impact) }\end{array}$ \\
\hline Mental health & $31.2,<0.0001$, Yes & $77.9,<0.0001$, Yes & 4.6, 0.033, Yes & $\begin{array}{l}\text { Reduces (depression lessens } \\
\text { impact) }\end{array}$ \\
\hline Physical component score & $1.0,0.315$, No & $45.9,<0.0001$, Yes & $0.1,0.812, \mathrm{No}$ & No impact \\
\hline Mental component score & $38.8,<0.0001$, Yes & $69.3,<0.0001$, Yes & 9.9, 0.002, Yes & $\begin{array}{l}\text { Reduces (depression lessens } \\
\text { impact) }\end{array}$ \\
\hline
\end{tabular}

CES-D10 Center for Epidemiologic Studies depression scale 10-item short form, CNS-LS Center for Neurologic Study lability scale, $P B A$ pseudobulbar affect, $S F$-36 36-item short form health survey

${ }^{a}$ Depression defined by score of 10 or greater on the CES-D 10

${ }^{b}$ The first number in each cell is the F value, followed by the probability of larger F value, and whether the impact of the factor SF-36 item score is significant (yes/no)

${ }^{c}$ Assesses whether PBA group assignment had a significant impact (main effect) on SF-36 score

${ }^{\mathrm{d}}$ Assesses whether the presence of depression (CES-D10 > 10) had a significant impact (main effect) on SF-36 score

${ }^{\mathrm{e}}$ Indicates whether there is significant interacting effect of depression on the effect of PBA group assignment

${ }^{\mathrm{f}}$ Indicates whether assignment to the PBA group has an effect to increase or reduce the SF-36 item score. If an interaction is present, the statement in parentheses indicates the effect of the interaction, i.e., whether the presence of depression augments or reduces the impact of PBA group assignment on the SF-36 item score

the previous week including frustration (48\% vs. 23\%), feeling like a burden to those around them (35\% vs. $13 \%)$, irritation (34\% vs. $15 \%)$, feeling isolated (30\% vs. $9 \%)$, and anger (26\% vs. $12 \%)(P<0.05$ for all $)$.

The survey also asked PBA group respondents (no controls) a series of customized questions specific to the frequency and severity of involuntary laughing and crying episodes and the degree to which these episodes were burdensome or disrupted specific life activities. PBA group respondents were first asked, "Have you [has the patient you care for] experienced involuntary episodes of crying and/or laughing that were exaggerated or even contrary to how you [they] felt at the time?" A total of 280 PBA 
group respondents $(70.7 \%$ weighted for relative proportions of the underlying conditions within the total US population) answered "yes" to this question (83.6\% [weighted] for respondents with a CNS-LS score $\geq 21$ ). These percentages are somewhat lower than the estimated diagnostic accuracy of a CNS-LS score of 13 or greater for PBA (as validated in patients with ALS and MS), but are not surprising given that some patients with PBA may have poor insight into their symptoms, and some patients and caregivers may not characterize their symptoms using this specific definition.

Of those acknowledging involuntary or exaggerated laughing/crying, 58\% said that the episodes were extremely (9\%), very $(16 \%)$, or somewhat $(33 \%)$ burdensome to them/their patient. The perception of burden correlated with laughing/crying episode frequency. In patients describing their episodes as extremely or very burdensome, mean episode frequencies for the past week were 8.8 for crying and 4.6 for laughing compared with 2.8 and 2.7, respectively, for respondents describing their episodes as somewhat or not burdensome $(P<0.05$ for both laughing and crying frequency). When these respondents were asked to indicate how much they agreed with certain statements surrounding their/their patient's laughing and crying episodes, given five possible response choices ranging from "strongly disagree" to "strongly agree", 60\% said ("somewhat" or "strongly agreed") that they/their patient feel(s) embarrassed when the

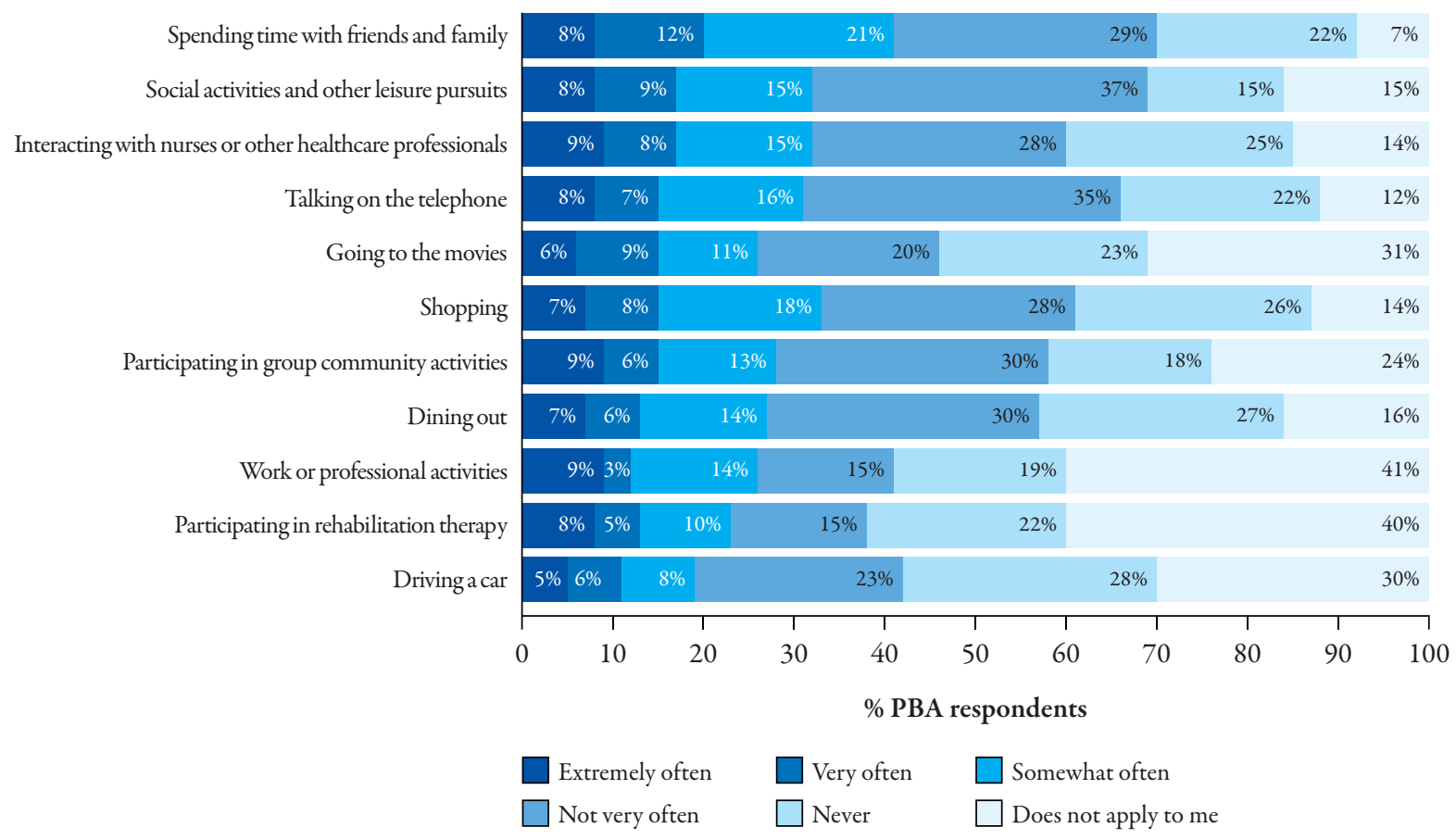

Fig. 3 Interference of laughing and crying episodes with patient function. Percentage of PBA group respondents selecting the given option in response to the following question, "Please indicate, how often you would say the episodes of involuntary crying and/or laughing interfere with your [your patient's] participation in each of the following activities. If you [your patient] do [does] not participate in an activity, please select 'Does not apply to me [him/her]." This question was completed only by PBA group respondents who also answered "yes" to the question, "Have you [has the patient you care for] ever experienced involuntary episodes of crying and/or laughing that were exaggerated or even contrary to how you [he/she] felt at the time?" $P B A$ pseudobulbar affect 


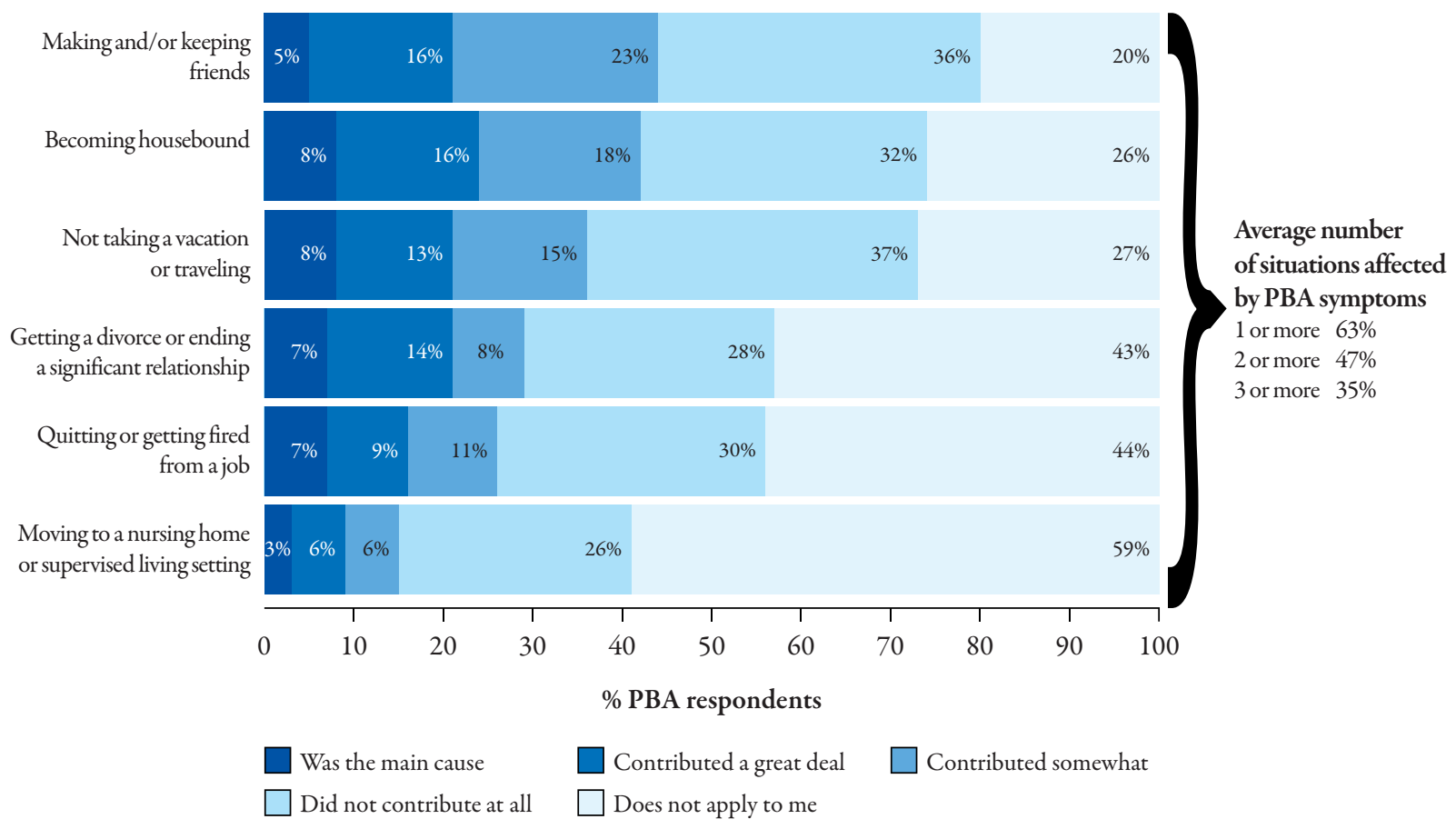

Fig. 4 Interference with life situations. Percentage of PBA group respondents selecting the given option in response to the following question: "To what extent have your [your patient's] involuntary episodes of laughing and/or crying ever contributed to the following life situations? Please select all situations that apply." This question was completed only by PBA group respondents who also answered "yes" to the question, "Have you [has the patient you care for] ever experienced involuntary episodes of crying and/or laughing that were exaggerated or even contrary to how you [he/she] felt at the time?" $P B A$ pseudobulbar affect

episodes occur, 58\% said that they/their patient feel(s) withdrawn from those around them, 51\% said that their/their patient's laughing/crying episodes are a problem for others, and $57 \%$ said that preventing the episodes would increase their/ their patient's QOL. Thirty-four percent of these PBA group patients and 38\% of these caregivers said that it was "very" or "extremely important" to them/their patient that the episodes of involuntary crying and/or laughing be treated.

PBA group respondents who acknowledged involuntary laughing and crying episodes were then asked to specify the degree to which the episodes interfered with participation in 11 social or instrumental activities; available response choices were "never," "not very often," "somewhat often," "very often," "extremely often," or "does not apply to me [him/her]."
From $19 \%$ to $41 \%$ of these respondents said that involuntary laughing or crying interfered at least somewhat often with each of the following activities: spending time with friends and family; social activities and other leisure pursuits; interacting with healthcare professionals; shopping; talking on the telephone; participating in group/community activities; dining out; work or professional activities; going to the movies; participating in rehabilitation therapy; and driving a car (Fig. 3). In addition, from $9.0 \%$ to $24.4 \%$ of these respondents said that involuntary episodes of laughing and/or crying contributed "a great deal" to or "were the main cause" of distressing life situations such as moving to a nursing home or supervised living (9\%), quitting or being fired from a job (16\%), getting a divorce or ending a 
significant relationship (21\%), or the patient's becoming housebound (24\%) (Fig. 4).

\section{DISCUSSION}

This survey was designed to measure the burden of PBA across a large community sample of patients with predisposing neurological disorders. The results from validated scales suggest that PBA is associated with significant impairment in overall health status and social and occupational functioning compared with controls with the same primary neurological conditions but without PBA. Involuntary episodes of laughing and/or crying affected patient-rated QOL and QOR and were associated with considerable psychological distress. In response to nonvalidated, customized questions, most PBA group respondents who acknowledged involuntary and inappropriate episodes of laughing and crying said they/their patient felt embarrassed by the episodes, withdrawn from those around them, and that preventing these episodes would improve their/their patient's QOL. Their responses to the customized questions suggest PBA can impact daily activities and social relationships, and in some cases, even contribute to such extreme events as becoming housebound, ending a significant relationship, or being placed in a nursing home.

Among the caregiver-specific measures, caring for a patient with presumed PBA (CNS-LS $\geq 13$ ) was associated with reduced caregiver work productivity and greater overall work impairment compared with caregivers for patients without presumed PBA (CNS-LS $<13$ ). Depression was common in caregivers $(>70 \%$ had CES-D10 scores $\geq 10$ ), and occurred with similar frequency in both groups.

No previously published studies, to the authors' knowledge, have sought to assess the burden associated with PBA across multiple domains and in a variety of underlying conditions. Calvert et al. [8] investigated psychosocial correlates of PBA in stroke survivors with and without "emotionalism". Patients with emotionalism were identified via the "House" criteria, which are composed of three questions asking whether patients had recently experienced excessive, unexpected, and uncontrollable crying or laughing episodes (essentially) consistent with PBA [29]. Stroke patients with "emotionalism" had significantly higher rates of simple depression, tension, social unease, irritability, other depression, general anxiety, lack of energy, loss of interest, and ideas of reference compared with stroke patients without "emotionalism". The finding of a significantly higher rate of depression in patients with emotionalism is consistent with the present survey, which found a significantly higher prevalence of depressive symptoms in PBA group patients compared with controls, including in the subgroup with stroke.

Strowd et al. [11] published a retrospective chart review that assessed PBA prevalence and association with mood disorders and QOL in patients with movement disorders $(n=269)$, including PD ( $n=168)$, essential tremor $(n=35)$, dystonia $(n=13)$, psychogenic movement disorders $(n=16)$, and other diagnoses $(n=28)$. As part of routine clinic assessments all patients had completed the CNS-LS, Beck depression inventory (BDI), and Parkinson's disease questionnaire (PDQ-39), a 39-question, validated instrument that assesses eight dimensions of health-related QOL, including emotional wellbeing $[11,30]$. In that study, patients with PBA had significantly higher (worse) scores on the BDI and PDQ-39 emotional well-being subscores compared with those without PBA regardless of whether a CNS-LS threshold of 13 or greater or 17 or greater was used to define PBA. The association between PBA and 
depression persisted even after removing BDI question 10 pertaining to episodes of crying. The relationship between PBA and depression in these patients is consistent with the CES-D10 scale results in our study, suggesting significantly higher rates of depression in PBA group patients overall compared with controls, including the subgroup with PD. The higher PDQ-39 emotional well-being subscores (including the items "depressed," "isolated and lonely," "weepy or tearful," "angry or bitter," "anxious," "worried about the future") [31] among the movement disorders patients in Strowd et al. [11] may also parallel our survey findings of increased depression, lower SF-36 mental component domain and summary scores, and increased psychological distress in PBA group patients versus controls.

Two other studies in patients with PD examined the relationship of PBA and depression; one $(n=131)$ found no association between Hamilton depression rating scale scores and a diagnosis of involuntary emotional expression disorder (IEED) [14]; while the other, which also included patients with other movement disorders ( $n=387$ PD patients; $n=332$ other movement disorders), did show a significant association of depression and PBA in the total study population [10]. Both of these studies also found greater functional impairment in the unified Parkinson's disease rating scale $\mathrm{ADL}$ section in patients with $\mathrm{PBA}$ (IEED); however, this association was considered more likely to be attributable to greater duration or severity of illness in these patients $[10,14]$.

Although previous studies have not examined differences in workplace function in patients with and without PBA, some findings may be relevant to the impaired work function observed in this survey. For example, patients with PBA have been shown to have reduced cognitive function in an MS population [32, 33] and in an ALS population [34], as well as reduced executive function in a stroke population [12], when compared with patients with the same underlying conditions but without PBA. Cognitive problems, in turn, have been correlated with unemployment in the MS population [35-37], and measures of executive dysfunction have been associated with unemployment in stroke patients [38]. In addition, a reported case study in a nonambulatory but full-time, employed patient with MS illustrates how the socially inappropriate and disruptive symptoms of PBA, such as "giggling or crying out during meetings," may have substantial adverse impacts on work function, independently of the other symptoms of the underlying disease [39]. Indeed, in this survey $16 \%$ of PBA group respondents reported that inappropriate episodes of laughing/crying were the main cause of or contributed a great deal to job loss.

Based on VAS scores, uncontrollable laughter, tearfulness, or anger affected patients' overall QOR with others to a significantly greater extent in the PBA group patients compared with controls. This result is consistent with PBA patient and caregiver ratings on customized questions in this survey regarding the specific impact of uncontrollable laughing/crying episodes on a range of day-to-day social activities and interpersonal interactions. Most remarkable of which are the $42 \%, 29 \%$, and $15 \%$ of respondents who reported that laughing/ crying episodes contributed at least "somewhat," and $24 \%, 21 \%$, and $9 \%$ who said that they contributed a "great deal," or were "the main cause" of the patient becoming housebound, getting a divorce or ending a significant relationship, or being placed in a nursing facility/supervised living. Few other studies have examined the impact of PBA episodes on social function. A study of 92 patients with TBI reported that those with pathological laughter or 
crying showed significantly greater impairment on social functioning exam scores [40] compared with those who did not have these symptoms [9]. Conversely, a matched case-control study $(n=24)$ of neurobehavioral correlates of pathological laughing and crying in patients with MS did not show differences between groups in the social dysfunction component of the general health questionnaire; however, patients in this sample were said to be severely disabled, and general health questionnaire social dysfunction items were commonly endorsed by both case and control groups in the study [32].

Interpretation of the survey results must consider several limitations. Although the authors attempted to adjust for differences in underlying disease severity and some demographic variables, these and a variety of other factors could still have affected survey results. To control for potential between-group differences in underlying disease severity, weighting factors were applied to the results based on respondent self-assessments. Patient/ caregiver assessments of overall disease severity were considered to be a valid severity measure based on positive correlations with the diseasespecific severity scale scores in four of the six evaluated disease states. That said, the simplistic range of responses (mild, moderate, severe) on global ratings may not have captured more subtle severity differences that may exist among patients or broadly and reliably reflect more objective disease severity measures. The extent to which the presence of PBA, depression, or other associated symptoms may have impacted self-assessed severity is not known.

Likewise, demographic weightings were used to adjust for between-group differences in age and gender; however, no adjustments were made to account for the potential influence of other demographic factors, such as patient/ caregiver education, socioeconomic status, the patient's living situation, the presence of a caregiver, or the patient's relationship to the caregiver. The relative proportion of caregivers and patient respondents was not uniform among the individual disease groups, and ALS and $\mathrm{AD}$ patients were underrepresented in the sample, precluding a thorough evaluation of potential differences in survey measures in these disease states. In addition, the use of caregivers as patient proxies, although allowing a more representative patient sample, may have introduced additional bias.

To improve generalizability and ensure that the contribution of PBA responses from individual disease groups reflected the population at large, the overall results were additionally weighted to reflect the relative proportions of the primary neurological conditions, and estimated prevalence of PBA within each condition, in the total US population.

Another important limitation is that patients in this trial were not required to have been clinically diagnosed with PBA to be included in the survey, with determination of PBA based exclusively on a CNS-LS score of 13 or greater. While the CNS-LS had been validated as a measure of PBA symptoms in ALS and MS at the time of this survey, and is frequently used as a screening instrument, it is not diagnostic for PBA. Subsequent to this survey, a validation study was published in patients with PD, showing a sensitivity of 0.93 and a specificity of 0.51 for symptoms or diagnosis of IEED (a diagnosis similar to PBA) with a CNS-LS threshold of 11 ; the CNS-LS showed poor discriminant validity for IEED in these patients, due to high correlation with depressive symptoms [41]. The CNS-LS has not been validated in patients with other underlying neurological conditions. The CNS-LS threshold of 13 used to determine the PBA group in this survey was based on the 
validation studies in ALS and MS and meant to ensure the inclusion of patients with more moderate PBA symptoms. The lower specificity of the CNS-LS in MS and PD suggests that at least some patients were assigned to the PBA group who did not have clinical PBA. It is noteworthy, however, that $71 \%$ of PBA group respondents did answer "yes" to the survey question, "Have you [has the patient you care for] experienced involuntary episodes of crying and/or laughing that were exaggerated or even contrary to how you [they] felt at the time?" consistent with the presence of PBA; another $10 \%$ answered this question as "unsure" and 19\% said "no," indicating a potential lack of PBA diagnosis or at least a lack of awareness of PBA symptoms. Future studies should consider use of a "gateway" question such as this one or short diagnostic interview to ascertain PBA.

As noted above, a positive association between CNS-LS scores and depressive symptoms has been observed in patients with ALS and PD $[11,15,41]$, and the prevalence of depression was also significantly higher in PBA group patients (87\%) than controls (56\%) in this study. Depression can impact burden measures such as the SF-36 [28]. To address this limitation, a post hoc MANOVA analysis was conducted. The results suggested that both depression and PBA group assignment separately impacted SF-36 domain and component scores, with depression exerting a significant negative impact on all SF-36 domains, PBA group assignment exerting a significant negative impact on all SF-36 domains except bodily pain and the physical component score, and an interaction of depression and PBA group assignment lessening the impact of PBA group assignment on the SF-36 subscales of role physical, role emotional, mental health, and the mental health component scores. While it may seem counterintuitive that an interaction of depression with PBA group assignment would serve to lessen the negative impact of PBA group assignment on SF-36 subscales, this could perhaps be explained by potential difficulties of the CNS-LS to discriminate depression reliably from PBA, i.e., some depressed patients may have been categorized as PBA when, in fact, they only had depression.

The association of PBA group assignment and depression is also of clinical relevance in that patients with PBA may be misdiagnosed with depression on the basis of their PBA episodes [41, 42]. Indeed, among the minority of survey respondents who had spoken with their physicians and received a diagnosis for their involuntary episodes of laughing and/or crying ( $n=63$ ), 23\% were diagnosed with depression, and another 5\% with bipolar disorder; $1.1 \%$ were given a diagnosis of either PBA or emotional lability. In the process of testing, evaluating and validating the CNS-LS scale in ALS patients, Moore et al. [15] found there was a significant relationship between the crying/tearfulness subscale of the CNS-LS and BDI scores; however, variability in depression scores accounted for only $6 \%$ of the variance in CNS-LS scores. While depression and PBA may occur alone or together, it is important to evaluate patients with crying episodes to ensure that neither is overlooked.

Finally, the nonlongitudinal design of this survey did not allow evaluation as to whether difficulties with social and occupational function develop coincidently with the emergence of PBA symptoms or are alleviated with PBA treatment.

\section{CONCLUSION}

This study found that patients and primary caregivers of patients with presumed PBA (CNS-LS $\geq 13$ ) have a significantly increased burden of illness compared with patients and caregivers of patients with the same underlying 
neurological conditions but without PBA. PBA group patients showed significantly greater impairment on measures of general health status, occupational and social function, and a significantly higher incidence of depressive symptoms than controls, both across and within primary neurological disease groups. Inappropriate laughing/crying episodes were associated with feelings of embarrassment, frustration, and the potential for withdrawal and isolation. Additional research is warranted to confirm these findings, further characterize the prevalence and impact of PBA, and improve awareness and recognition of this disorder.

\section{ACKNOWLEDGMENTS}

Susan Work of Avanir Pharmaceuticals, Inc., coordinated the design and implementation of the survey. Ryan Arnold, DO, previously of Avanir Pharmaceuticals, Inc., and Shawn Wade, PhD, previously of Harris Interactive Inc. (HI), contributed to the survey and research design. Kerri Miller and Jim Llewellyn of HI and Dan Glass and Shawn Wade, previously of HI, helped in the statistical analysis of the results. Bonnie Dean of Cerner Health Insights, Beverly Hills, California, USA, provided assistance with statistical interpretation. The authors meet the criteria for authorship as recommended by the International Committee of Medical Journal Editors (ICMJE), are fully responsible for all content and editorial decisions, and were involved at all stages of manuscript development. Editorial assistance in preparing the manuscript for publication was provided by The Curry Rockefeller Group, LLC, Tarrytown, New York, USA. Support for this assistance was funded by Avanir Pharmaceuticals, Inc. Dr. Formella is the guarantor for this article, and takes responsibility for the integrity of the work as a whole.
Conflict of Interest. J. Colamonico has nothing to disclose. A. Formella has received personal compensation for activities with Avanir Pharmaceuticals, Inc., as an employee. W. Bradley has received research grants from Avanir Pharmaceuticals, Inc.

Open Access. This article is distributed under the terms of the Creative Commons Attribution Noncommercial License, which permits any noncommercial use, distribution, and reproduction in any medium, provided the original author(s) and source are credited.

\section{REFERENCES}

1. Dark FL, McGrath JJ, Ron MA. Pathological laughing and crying. Aust NZ J Psychiatry. 1996;30:472-9.

2. Schiffer R, Pope LE. Review of pseudobulbar affect including a novel and potential therapy. J Neuropsychiatry Clin Neurosci. 2005;17:447-54.

3. Wortzel HS, Oster TJ, Anderson CA, Arciniegas DB. Pathological laughing and crying: epidemiology, pathophysiology and treatment. CNS Drugs. 2008;22:531-45.

4. Arciniegas DB, Topkoff J. The neuropsychiatry of pathologic affect: an approach to evaluation and treatment. Semin Clin Neuropsychiatry. 2000;5:290-306.

5. Work S, Colamonico JA, Bradley WG, Kaye RE. Pseudobulbar affect: an under-recognized and undertreated neurological disorder. Adv Ther. 2011;28:586-601.

6. Wilson SAK. Some problems in neurology. II: pathological laughing and crying. J Neurol Psychopathol. 1924;4:299-333.

7. Davison C, Kelman H. Pathologic laughing and crying. Arch Neurol Psychiatry. 1939;42:595-643.

8. Calvert T, Knapp P, House A. Psychological associations with emotionalism after stroke. J Neurol Neurosurg Psychiatry. 1998;65:928-9.

9. Tateno A, Jorge RE, Robinson RG. Pathological laughing and crying following traumatic brain injury. J Neuropsychiatry Clin Neurosci. 2004;16:426-34. 
10. Siddiqui MS, Fernandez HH, Garvan CW, et al. Inappropriate crying and laughing in Parkinson disease and movement disorders. World J Biol Psychiatry. 2009;10:234-40.

11. Strowd RE, Cartwright MS, Okun MS, Haq I, Siddiqui MS. Pseudobulbar affect: prevalence and quality of life impact in movement disorders. J Neurol. 2010;257:1382-7.

12. Tang WK, Chen Y, Lam WW, et al. Emotional incontinence and executive function in ischemic stroke: a case-controlled study. J Int Neuropsychol Soc. $2009 ; 15: 62-8$.

13. Choi-Kwon S, Kim JS. Poststroke emotional incontinence and decreased sexual activity. Cerebrovasc Dis. 2002;13:31-7.

14. Petracca GM, Jorge RE, Ación L, Weintraub D, Robinson RG. Frequency and correlates of involuntary emotional expression disorder in Parkinson's disease. J Neuropsych Clin Neurosci. 2009;21:406-12.

15. Moore SR, Gresham LS, Bromberg MB, Kasarkis EJ, Smith RA. A self-report measure of affective lability. J Neurol Neurosurg Psychiatry. 1997;63:89-93.

16. Smith RA, Berg JE, Pope LE, Callahan JD, Wynn D, Thisted RA. Validation of the CNS emotional lability scale for pseudobulbar affect (pathological laughing and crying) in multiple sclerosis patients. Mult Scler. 2004;10:1-7.

17. Brooks BR, Thisted RA, Appel SH, et al; AVP-923 ALS Study Group. Treatment of pseudobulbar affect in ALS with dextromethorphan/quinidine: a randomized trial. Neurology. 2004;63:1364-70.

18. Panitch HS, Thisted RA, Smith RA, et al; Pseudobulbar Affect in Multiple Sclerosis Study Group. Randomized, controlled trial of dextromethorphan/ quinidine for pseudobulbar affect in multiple sclerosis. Ann Neurol. 2006;59:780-7.

19. Pioro EP, Brooks BR, Cummings J, et al; Safety, Tolerability, and Efficacy Results Trial of AVP-923 in PBA Investigators. Dextromethorphan plus ultra low-dose quinidine reduces pseudobulbar affect. Ann Neurol. 2010;68:693-702.

20. Faul M, Xu L, Wald MM, Coronado VG. Traumatic brain injury in the United States: emergency department visits, hospitalizations and deaths 2002-2006. Overview, page 13. Atlanta: Centers for Disease Control and Prevention, National Center for Injury Prevention and Control; 2010. Available at: http://www.cdc.gov/traumaticbraininjury/pdf/ blue_book.pdf Accessed Aug 82012.
21. Ware JE Jr., Sherbourne CD. The MOS 36-item short-form health survey (SF-36). I. Conceptual framework and item selection. Med Care. 1992;30:473-83.

22. Reilly MC, Zbrozek AS, Dukes EM. The validity and reproducibility of a work productivity and activity impairment instrument. Pharmacoeconomics. 1993;4:353-65.

23. Vitaliano PP, Russo J, Young HM, Becker J, Maiuro $\mathrm{RD}$. The screen for caregiver burden. Gerontologist. 1991;31:76-83.

24. Andresen EM, Malmgren JA, Carter WB, Patrick DL. Screening for depression in well older adults: evaluation of a short form of the CES-D (Center for Epidemiologic Studies Depression Scale). Am J Prev Med. 1994;10:77-84.

25. Cedarbaum JM, Stambler N. Performance of the amyotrophic lateral sclerosis functional rating scale (ALSFRS) in multicenter clinical trials. J Neurol Sci. 1997;152:S1-9.

26. Schwab RS, England AC. Projection technique for evaluating surgery in Parkinson's disease. In: Gillingham FJ, Donaldson IML, eds. Third Symposium on Parkinson's Disease; May 20-22 1969, Edinburgh, UK.

27. Lawton MP, Brody EM. Assessment of older people: self-maintaining and instrumental activities of daily living. Gerontologist. 1969;9:179-86.

28. Guajardo V, Souza BP, Henriques SG, et al. Loss of interest, depressed mood and impact on the quality of life: cross sectional survey. BMC Pub Health. 2011;11:826.

29. House A, Dennis M, Molyneux A, Warlow C, Hawton K. Emotionalism after stroke. BMJ. 1989;298:991-4.

30. Jenkinson C, Peto V, Fitzpatrick R, Greenhall R, Hyman N. Self-reported functioning and well-being in patients with Parkinson's disease: comparison of the short-form Health Survey (SF36) and the Parkinson's Disease Questionnaire (PDQ-39). Age Ageing. 1995;24:505-9.

31. Hagell P, Nygren C. The 39 item Parkinson's disease questionnaire (PDQ-39) revisited: implications for evidence based medicine. J Neurol Neurosurg Psychiatry. 2007;78:1191-8.

32. Feinstein A, Feinstein K, Gray T, O'Connor P. Prevalence and neurobehavioral correlates of pathological laughing and crying in multiple sclerosis. Arch Neurol. 1997;54:1116-21. 
33. Feinstein A, O'Connor P, Gray $\mathrm{T}$, Feinstein K. Pathological laughing and crying in multiple sclerosis: a preliminary report suggesting a role for the prefrontal cortex. Mult Scler. 1999;5:69-73.

34. McCullagh S, Moore M, Gawel M, Feinstein A. Pathological laughing and crying in amyotrophic lateral sclerosis: an association with prefrontal cognitive dysfunction. J Neurol Sci. 1999;169:43-8.

35. Edgley K, Sullivan MJ, Dehoux E. A survey of multiple sclerosis: part 2. Determinants of employment status. Can J Rehabil. 1991;4:127-32.

36. Julian LJ, Vella L, Vollmer T, Hadjimichael O, Mohr DC. Employment in multiple sclerosis: exiting and reentering the work force. J Neurol. 2008;255:1354-60.

37. Morrow SA, Drake A, Zivadinov R, Munschauer F, Weinstock-Guttman B, Benedict RH. Predicting loss of employment over three years in multiple sclerosis: clinically meaningful cognitive decline. Clin Neuropsychol. 2010;24:1131-45.
38. Ownsworth T, Shum D. Relationship between executive functions and productivity outcomes following stroke. Disabil Rehabil. 2008;30: 531-40.

39. Lantz MS. Pathologic laughing and crying in multiple sclerosis. Clin Geriatr. 2005;13:14-7.

40. Starr LB, Robinson RG, Price TR. Reliability, validity, and clinical utility of the social functioning exam in the assessment of stroke patients. Exp Aging Res. 1983;9:101-6.

41. Phuong L, Garg S, Duda JE, Stern MB, Weintraub D. Involuntary emotional expression disorder (IEED) in Parkinson's disease. Parkinsonism Relat Disord. 2009;15:511-5.

42. Cummings JL, Arciniegas DB, Brooks BR, et al. Defining and diagnosing involuntary emotional expression disorder. CNS Spectrums. 2006;11:1-7. 